\title{
Migrainous headache and gliotic thalamic lesion after treated neuroblastoma in childhood
}

\author{
Smaranda Antonia Nita1,2, Raluca Ioana Teleanu ${ }^{1,2}$, Diana Epure' ${ }^{1}$, Ovidiu Bajenaru ${ }^{1,3}$ \\ 1"Carol Davila" University of Medicine and Pharmacy, Bucharest, Romania \\ 2Department of Pediatric Neurology, "Dr. Victor Gomoiu” Children's Hospital, Bucharest, Romania \\ ${ }^{3}$ Neurology Department, Emergency University Hospital, Bucharest, Romania
}

\begin{abstract}
Objective. This article aims to bring into attention the challenges of diagnosing a case of migraine in a child with a history of neuroblastoma and an associated finding of gliotic thalamic lesion on the cerebral MRI.

Materials and methods. The patient underwent a thorough neurological examination, followed by paraclinical tests: blood workup, cerebral MRI with TOF (time-of-flight) angiography, electroencephalogram (EEG), carotid and vertebral arterial Doppler examination, trancranial Doppler examination, cardiac and abdominal ultrasounds, psychological evaluation.

Results. The neurological examination was normal, except for right sided post-surgical Claude Bernard-Horner's syndrome. His blood workup, ultrasounds, EEG, as well as vascular and transcranial Doppler examinations were within normal limits. His cerebral MRI showed a right anterior thalamic ischemic gliotic lesion.

Discussions. Neuroblastoma is a common tumor in childhood and its treatment involves surgery, chemotherapy and radiotherapy. A patient with a history of neuroblastoma who has new onset headache has to be evaluated for a possible connection to his pathological background and to the treatment associated with it. A new malignancy and long term side effects of radiotherapy or chemotherapy must be taken into consideration.

Conclusions. The headache episodes were considered as migraine without aura. The article aims to bring into attention the difficulties of diagnosing migraine in children, especially in patients with a neurological history.
\end{abstract}

Keywords: neuroblastoma, child, headache, migraine, thalamic lesion

\section{INTRODUCTION}

Neuroblastoma is the most common extracranial tumor in childhood and it is a malignancy of the sympathetic nervous system, arising from neuroblasts. Its treatment is based on surgical ablation, chemotherapy, with added radiotherapy, depending on the stage of the disease (1).

\section{Central nervous system lesions after cancer treatment}

Lesions of the central nervous system have been documented after radiotherapy for different malignances, both in children and in adults. The most common forms of pathologic occurrences after radiotherapy are radiation necrosis, white matter injury, leukoencephalopathy, radiation induced telan- giectasias. Chemotherapy can also lead to white matter lesions and leukoencephalopathy, both alone and in combination with radiotherapy, as well as posterior reversible encephalopathy syndrome and cerebral volume loss. The combination of radiotherapy and chemotherapy can cause necrotizing leukoencephalopathy and mineralizing microangipathy (2). Ischemic stroke is cited as a complication of antiangiogenic therapy (3). Basal ganglia and, rarely, thalamic calcifications have been described after both radio- and chemotherapy (4).

\section{Impact of post-treatment lesions on clinical and cognitive outcome}

Depending on the character and the magnitude of the lesions, they can have catastrophic effects on the clinical outcome of the patients or they can 
seemingly leave no deficits with respect to motor functions while negatively affecting the cognitive profile. One of the studies describes that part of the pediatric patients who underwent craniospinal irradiation and high-dose chemotherapy (protocol including cisplatin, vincristine, amifostine, cyclophosphamide) for medulloblastoma and primitive neuroectodermal tumor developed a decrease in their long-term cognitive abilities (IQ, Wechsler Individual Achievement Test), in the presence of white matter lesions in the pons and cerebellum (5).

Other authors describe a long-term negative impact of standard dose systemic chemotherapy on cognitive functioning in a subgroup of adult patients with breast cancer and lymphoma. The study used neuropsychologic tests such as Center for Epidemiological Study-Depression, Spielberger State-Trait Anxiety Inventory, and Fatigue Symptom Inventory (6).

Other studies plead for a more thorough assessment of the cognitive functioning of cancer survivors, besides neuropsychological testing, which includes EEG, MRI and fMRI (7), to unlock different substrates of the so called "chemobrain", a term that has become a well known clinical follow-up phenomenon manifesting in a subgroup of longterm cancer survivors as different degrees of cognitive impairment (8).

Studies show that chemotherapy alone does not affect the cognitive function in children but has a high likelihood of negatively influencing it if combined with craniospinal radiotherapy for brain tumors (9).

\section{OBJECTIVE}

This article aims to bring into attention the challenges of diagnosing a case of migraine in a child with a history of neuroblastoma and an associated finding of gliotic thalamic lesion on the cerebral MRI.

\section{MATERIALS AND METHODS}

We present the case of a ten year old male patient, with no significant family or personal history and normal motor and cognitive development, who at the age of one year and eight months was diagnosed with a posterior thoracic mass extending into the mediastinum, diagnosed by chest $\mathrm{CT}$ and postoperatory histopathologically confirmed as neuroblastoma. There was local periesophageal adipose tissue infiltration, as well as mediastinal lymph node metastasis. He underwent chemotherapy with doxorubicin, vincristine and cyclophosphamide under oncologic monitoring, with a favorable evolution. There was no evidence of distance metastasis or other tumor sites on seriated imaging (cerebral, spine, abdominal and pelvic $\mathrm{CT}$ and MRI). $\mathrm{He}$ underwent cerebral MRI two years post-tumor resection and chemotherapy, which was normal.

Postoperatory, the child developed right sided Claude Bernard-Horner syndrome, which was confirmed clinically and by sympathetic skin response study. There was no evidence of brachial plexus injury.

After approximately eight symptom-free years, the child was admitted to our Department for episodic headache localized to the right supraorbital region, accompanied by vomiting, each episode lasting up to an hour and being calmed down by sleep or acetaminophen. The frequency of the episodes was of one - two episodes per month for about one year, increasing to up to one - two episodes per week, prior to admission.

\section{RESULTS}

The patient's neurological examination was normal, except for right sided Claude Bernard-Horner's syndrome. The psychological evaluation revealed normal intelligence but marked emotional instability.

The usual blood workup was normal and the heart and abdominal ultrasound examinations were normal. His wake electroencephalogram (EEG) was normal for age.

Native cerebral MRI with TOF (time-of-flight) angiography was performed, which revealed normal cerebrospinal fluid spaces, no evidence of arterial stenosis or malformations and a normal brain parenchyma, with the exception of a right anterior thalamic ischemic gliotic lesion, measuring approximately $0.4 \mathrm{~cm}$, with no restricted diffusion and no perilesional edema (Figures 1,2).

Carotid and vertebral arterial Doppler revealed normal vessel walls, as well as normal blood velocity, bilaterally (Figures 3, 4, 5). 


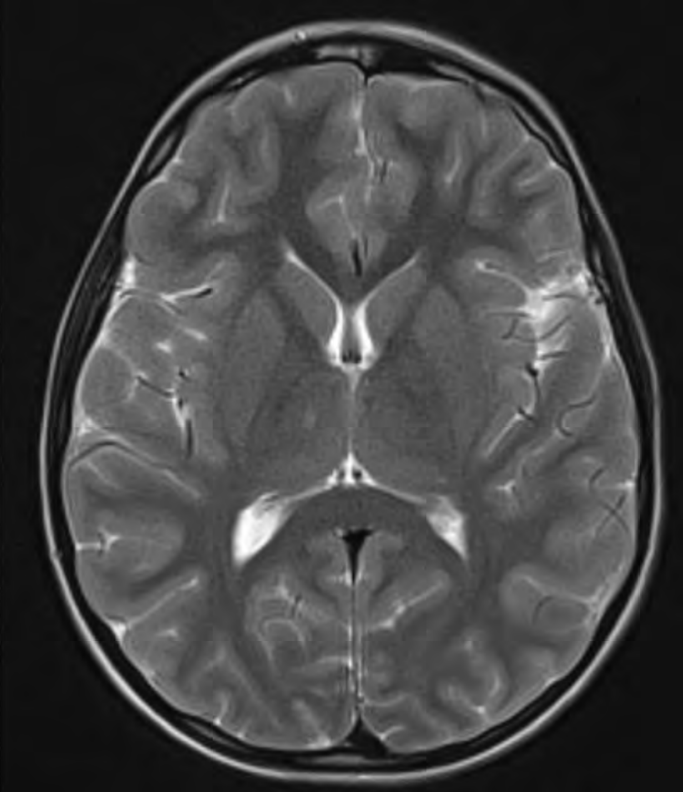

FIGURE 1. Axial T2-weighted MR image, right hyperintense thalamic lesion

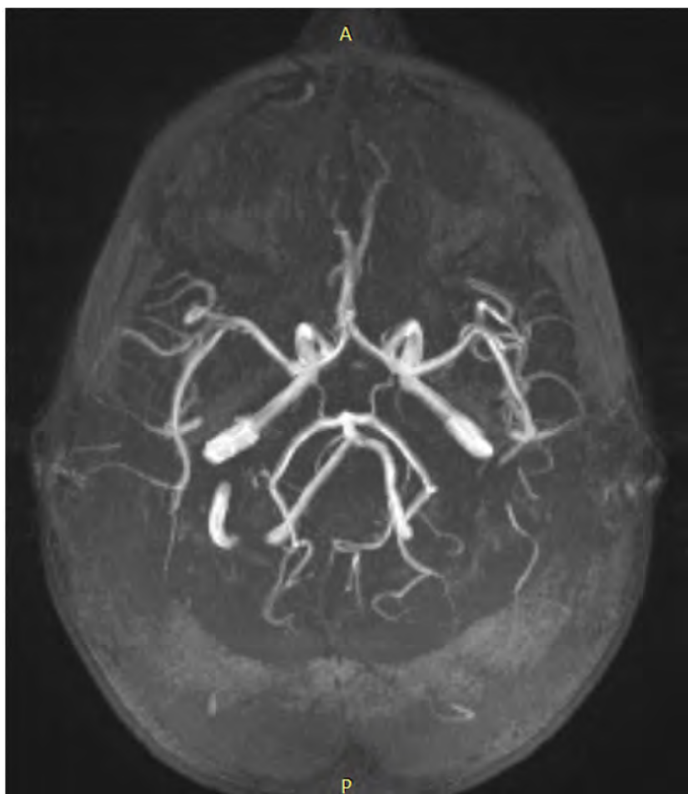

FIGURE 2. TOF angiography, normal aspect

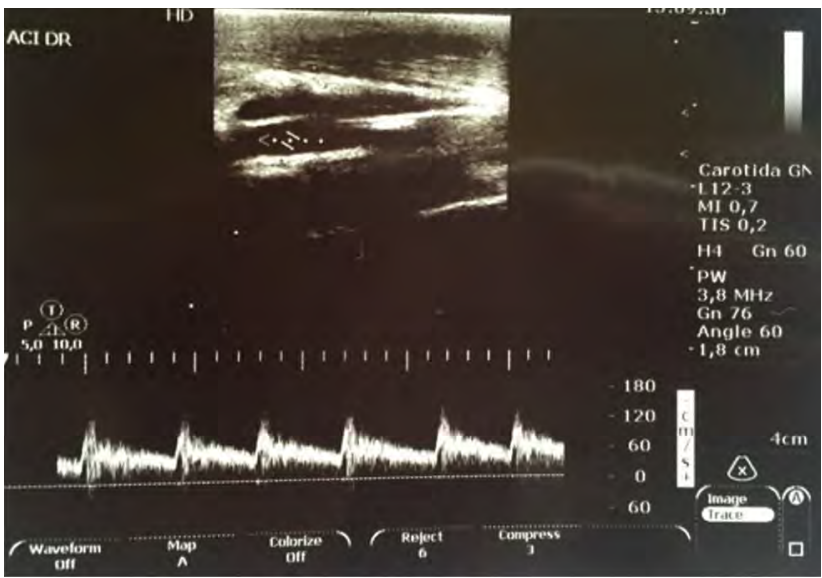

FIGURE 3. Cervical Doppler examination, right internal carotid

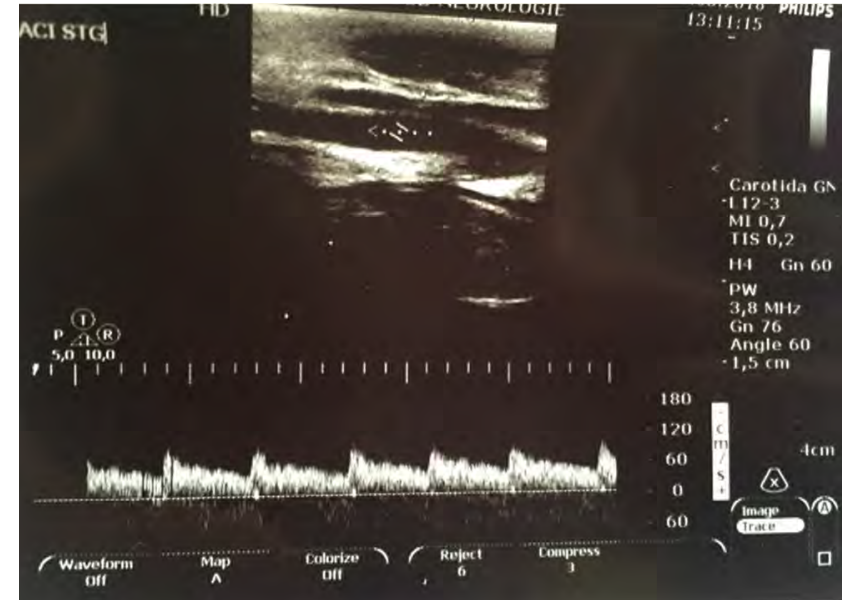

FIGURE 4. Cervical Doppler examination, left internal carotid

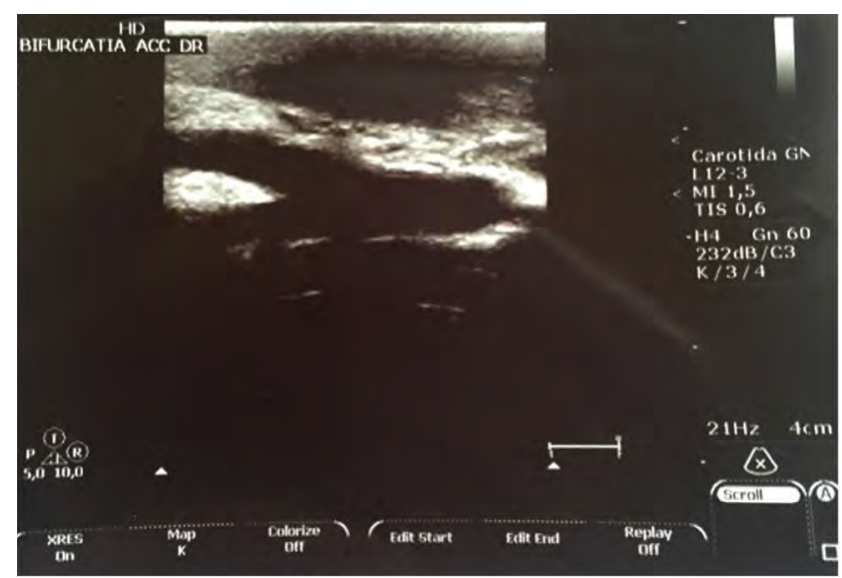

FIGURE 5. Cervical Doppler examination, right common carotid

Transcranial Doppler examination revealed symmetric blood flow, indicating no unilateral cerebral blood vessel related pathology.

The headache episodes responded well to abortive therapy with acetaminophen and their frequency decreased significantly after lifestyle changes, with a well defined sleep schedule and avoiding electronic devices.

\section{DISCUSSIONS}

Given the patient's symptoms and laboratory tests, the thalamic gliotic lesion was considered in light of his history of neuroblastoma. Although the patient has not received radiotherapy, the literature was reviewed for a possible delayed adverse reaction to chemotherapy, for which no arguments were found. The supraorbital pain described by the patient seemed not to be correlated to the thalamic lesion although a thalamic syndrome was taken into consideration. 


\section{CONCLUSIONS}

The headache episodes were considered as migraine without aura, considering the clinical features and the evolution and response to medication and non-pharmacological treatment. The article aimed to bring into attention the difficulties of di-

\section{REFERENCES}

1. National Cancer Institute, Neuroblastoma Treatment, https://www. cancer.gov/types/neuroblastoma/hp/neuroblastoma-treatmentpdq\#link/_760

2. Mamlouk MD, Handwerker J, Ospina J et al. Neuroimaging Findings of the Post-Treatment Effects of Radiation and Chemotherapy of Malignant Primary Glial Neoplasms. The Neuroradiology Journal, 2013;26(4):396-412

3. Fraum TJ, KreisI TN, Sul J. Ischemic stroke and intracranial hemorrhage in glioma patients on antiangiogenic therapy. J. neurooncolog., 2011;105(2):281-289

4. Dimitri Renard D, Castelnovo G, Campello $C$ et al. Thalamic Lesions: A Radiological Review. Behavioural Neurology, 2014; 2014:154631

5. Fouladi M, Chintagumpala M, Laningham FH et al. White Matter Lesions Detected by Magnetic Resonance Imaging After Radiothera- agnosing migraine in children, especially in patients with a neurological history.

\section{Acknowledgments}

Acknowledgments to Dr. Antochi F., Emergency University Hospital Bucharest, Neurology Department, for Doppler examination of the patient.

Conflict of interest: none declared Financial support: none declared

py and High-Dose Chemotherapy in Children With Medulloblastoma or Primitive Neuroectodermal Tumor. J Clin Oncol, 2004;22:45514560

6. Ahles TA, Saykin AJ, Furstenberg CT et al. Neuropsychologic impact of standard-dose systemic chemotherapy in long-term survivors of breast cancer and lymphoma. J Clin Oncol, 2002,15;20(2):485-493

7. Scherling CS, Smith A. Opening up the Window into "Chemobrain": A Neuroimaging Review, Sensors, 2013;13(3):3169-3203

8. Kaiser J, Bledowski C, Dietrich J. Neural correlates of chemotherapyrelated cognitive impairment. Cortex, 2014:54:33-50

9. Askins MA, Moore BD. Preventing Neurocognitive Late Effects in Childhood Cancer Survivors. Journal of child neurology, 2008;23(10):1160-1171 